\title{
El Papel de la Adaptación como Generadora de Satisfacción Laboral en Médicos de Salud Ocupacional
}

\section{THE ROLE OF ADAPTATION AS GENERATOR OF JOB SATISFACTION IN OCCUPATIONAL HEALTH DOCTORS}

\author{
Maria del Carmen López-García', María de los Ángeles Aguilera-Velasco², Diemen Darwin Delgado-García ${ }^{3}$ \\ 1. Escuela Nacional de Medicina y Homeopatía, Instituto Politécnico Nacional, México. Centro Universitario de Ciencias de la Salud, Universidad de \\ Guadalajara, México. \\ 2. Centro Universitario de Ciencias de la Salud, Universidad de Guadalajara, México. \\ 3. Profesor honorifico de Centro Universitario de Ciencias de la Salud, Universidad de Guadalajara, México.
}

\begin{abstract}
RESUMEN
Objetivo: analizar la construcción teórica del concepto de satisfacción laboral a través de la percepción de la adaptación al puesto de trabajo en médicos de salud ocupacional. Método: se aplicó la teoría fundamentada, utilizando el muestreo teórico propositivo hasta la saturación teórica. La muestra se integró con diez médicos dedicados a la salud ocupacional en la ciudad de México, durante el 2014. Se aplicaron entrevistas a profundidad y los datos obtenidos fueron analizados con el software Atlas-ti, dando como resultado códigos que se integraron en categorías, con las cuales se generó la teoría. Resultados: Mis motivos de elección, Cambié de vida, y Oportunidades y proyectos fueron las categorías emergentes con las cuales se integró la dimensión de Adaptación al puesto de trabajo como elemento básico de la construcción teórica de la satisfacción laboral de los médicos de salud ocupacional. Conclusiones: La satisfacción laboral en los médicos de salud ocupacional se presenta conforme se da su adaptación al puesto de trabajo, pues no existe desde el inicio de su vida laboral en el área, sino que se va generando conforme van aprendiendo y cambiando de actitud con respecto al trabajo.
\end{abstract}

(López-García M, Aguilera-Velasco M, Delgado-García D, 2015. El Papel de la Adaptación como Generadora de Satisfacción Laboral en Médicos de Salud Ocupacional. Cienc Trab. Sep-Dic; 17 [54]: 188-192).

Palabras clave: SATISFACCIÓN LABORAL, ADAPTACIÓN AL PUESTO DE TRABAJO, SALUD EN EL TRABAJO, MÉDICOS, TEORÍA FUNDAMENTADA.

\section{ABSTRACT}

Objective: to analyze the theoretical construction of job satisfaction's concept through the adaptation to the job's perception in occupational health physicians. Method: it was applied the grounded theory, using proactive until theoretical saturation theoretical sampling. The sample was composed of ten physicians dedicated to occupational health in Mexico city, during the 2014. In-depth interviews were applied and the data obtained were analyzed with the software Atlas-ti, giving as a result: codes, which were integrated into categories, with which the theory was generated. Results: My reasons for choice, I changed my life, and Opportunities and projects were the emerging categories with which joined the dimension of Adaptation to the post, as a basic element of the theoretical construction of the job satisfaction of occupational health physicians. Conclusions: Job satisfaction in occupational health physicians is presented as its adaptation to the job. It did not exist since the beginning of their working lives in the area, but it was generated as they learned and changed the attitude towards the work.

Keywords: JOB SATISFACTION, ADAPTATION TO THE POST, OCCUPATIONAL HEALTH, PHYSICIANS, GROUNDED THEORY

\section{INTRODUCCIÓN}

Los médicos que se dedican a la Salud Ocupacional en las empresas, según el Código Internacional de Ética para los Profesionales de la Salud Ocupacional (CISO), son aquellos que "en el ejercicio de su profesión, desempeñan tareas, proveen servicios o están involucrados en una práctica de seguridad y salud ocupacional”. ${ }^{1}$ Su misión

\section{Correspondencia / Correspondence:}

Maria del Carmen López-García

Escuela Nacional de Medicina y Homeopatía,

Instituto Politécnico Nacional, México.

Guillermo Massieu Helguera 239, Fracc.

La Escalera, Ticomán, C.P. 07320, México, D.F.

Tel: 525551192057

e-mail: mc.lpzg@gmail.com

Recibido: 29 de Septiembre de 2015 / Aceptado: 14 de Octubre de 2015 es la de mantener en óptimo estado de salud al trabajador para asegurar la continuidad de la fuerza laboral y, por tanto, el éxito de una industria. Su labor tiene un carácter integral: asistencial, preventivo, rehabilitador y, en última instancia, curativo. Su ejercicio está ligado a los programas de seguridad e higiene de la empresa, en labor conjunta con un equipo multidisciplinario. ${ }^{2}$

Estos médicos deben adquirir y mantener competencias necesarias para ejercer sus obligaciones, y exigir las condiciones que les permitan llevar a cabo sus tareas, de acuerdo a las buenas prácticas y la ética profesional". ${ }^{1}$

Desgraciadamente, dichas competencias sólo se adquieren de manera muy superficial en las escuelas y facultades de medicina, aun cuando existen numerosos posgrados (desde diplomados hasta doctorados) que capacitan a los profesionales en el área. Algunos aprovechan esta oportunidad escolarizada de prepararse, pero otros sólo van aprendiendo sobre la marcha.

Por otra parte, si consideramos que, como dice Engels, "el trabajo ha creado al hombre" , todo trabajador debe realizarse y encontrar satis- 
facción en el desempeño de su trabajo. Más aun los médicos de Salud Ocupacional que laboran procurando que otros trabajen con bienestar. La satisfacción laboral es un concepto que ha sido ampliamente estudiado desde 1935. Peiró la define como la actitud o conjunto de actitudes desarrolladas por la persona hacia su situación de trabajo ${ }^{4}$; Locke, como un estado emocional positivo o placentero de la percepción subjetiva de las experiencias laborales del sujeto; y Muchinsky, como el grado de placer que el empleado obtiene de su trabajo. ${ }^{5}$

De acuerdo con Spector, la satisfacción laboral repercute en aspectos individuales y colectivos, como el desempeño laboral, la salud y la longevidad. ${ }^{6}$ Asimismo, Fernández afirma que la mayor satisfacción laboral mejora la productividad ${ }^{7}$; y Argüelles, Quijano y Sahui refieren que la satisfacción laboral es promotora de la calidad de vida laboral de los trabajadores y que, con base en ello, es factible establecer las estrategias necesarias para promover la productividad y el rendimiento óptimo de los empleados. ${ }^{8}$

En cuanto a sus repercusiones en la salud, se ha documentado que la falta de satisfacción laboral sumada a características propias de cada persona, actúan como condicionantes para la aparición de Síndrome de Burnout, enfermedad laboral que se presenta en profesionales expuestos a condiciones psicosociales desfavorables en su medio laboral. ${ }^{9}$

Y aun cuando no existen estudios sobre el tema en médicos dedicados a la salud ocupacional, sí los hay sobre satisfacción laboral en personal de salud y sus resultados la refieren deficiente. ${ }^{10-13}$

Abrahan afirma que, dentro de una organización, la satisfacción laboral se relaciona directamente con la experiencia de la persona y que esta experiencia se transforma en la percepción del trabajador. Dicha percepción culmina en un componente emocional que, finalmente, desemboca en la manera de actuar del individuo. ${ }^{14}$

Dicho en otras palabras, Aguilera-Velasco, Pérez, Pozos y Acosta afirman que el concepto de salud en el trabajo en los profesionales es una construcción tanto individual como social... su percepción puede comprenderse mejor contextualizada en el universo de creencias, valores y comportamientos del medio sociocultural. En el marco cultural de las representaciones, roles y expectativas, los profesionales configuran lo que cada uno entiende y vive como la salud en el trabajo y sobre ese significado atribuido resuelven cotidianamente cómo cuidar la salud de los trabajadores y cómo recuperarla cuando se ha deteriorado, siendo un proceso dinámico no sólo desde la propia experiencia personal sino también en relación con el contexto en que se desenvuelve. ${ }^{15}$

De manera complementaria, estos mismos autores en el estudio que realizaron explorando los significados culturales del concepto de salud en el trabajo, antes y después de un diplomado sobre salud ocupacional, encontraron que la cultura de la prevención se logra a través de un proceso de aprendizaje que incluye conocimientos sobre los riesgos laborales y la normatividad vigente. ${ }^{15}$ Luego, entonces, los médicos dedicados a la salud ocupacional deben aprender, "experimentar" y elaborar su concepción teórica de salud ocupacional para actuar conforme a ella y, finalmente, adaptarse a un entorno organizacional distinto de aquel para el que fueron formados. Según el Modelo de la Adaptación a través de la Ocupación, la persona se adapta o ajusta (crece y se desarrolla) a través del uso de (o participación en) diversas ocupaciones; de esta forma, la persona, en este caso, los médicos que laboran en las empresas, pueden adaptarse al entorno o adaptarlo a sus necesidades individuales o para su satisfacción. ${ }^{16}$

Por otra parte, la mayoría de las investigaciones relacionadas con el tema son de corte explicativo y buscan medir el grado de satis- facción y sus determinantes. ${ }^{10-13}$ Los estudios cualitativos son menos, y aportan la visión emic de los trabajadores, no sólo etiquetándolo como satisfactorio o no, sino ofreciendo otras perspectivas, como la de los oficiales de la construcción españoles que refieren que su trabajo es "satisfactorio, divertido y fantástico", ya que tienen variedad en las tareas y son reconocidos. ${ }^{17} \mathrm{Y}$ la del personal de enfermería británico que asegura que una mayor responsabilidad profesional, como la de prescribir medicamentos, aumenta el grado de estrés, pero también el de satisfacción laboral. ${ }^{18} \mathrm{Y}$ los sentimientos de desgaste y soledad que viven los cuidadores de pacientes con demencia en Colombia. ${ }^{19}$

En esta investigación se buscó analizar la construcción teórica del concepto de satisfacción laboral a través de la percepción de la adaptación al puesto de trabajo en médicos de salud ocupacional. Siendo la satisfacción laboral una actitud que surge del conocimiento y creencias que tienen los trabajadores de su trabajo, se consideró conducente realizar este estudio a través de la metodología cualitativa, que ofrece la alternativa de validar científicamente el conocimiento ordinario.

\section{MÉTODO}

Se eligió para abordar este estudio la metodología cualitativa de la teoría fundamentada. Y es que no sólo tiene la capacidad de generar teoria, sino también, de fundamentarla en los datos. ${ }^{20}$

El estudio se ubicó en la ciudad de México e incluyó el periodo comprendido entre agosto de 2013 y junio del 2015. El muestreo fue teórico propositivo, es decir, que los participantes se fueron eligiendo conforme a la necesidad de precisión y refinamiento de la teoría que se fue generando. Se consideraron criterios de inclusión: ser médicos de medianas o grandes empresas, con dedicación de 40 horas semanales a la Salud Ocupacional (SO) en empresas de la ciudad de México; llevar mínimo 2 años laborando en el área de SO; y que aceptaran participar en la investigación, mediante consentimiento informado.

\section{Características socio-laborales de los participantes}

Los casos se constituyeron por cinco médicos del sexo masculino y cinco del sexo femenino, 60\% casados, 30\% solteros y 10\% en unión libre. Sus edades tuvieron un rango de 26 a 59 años, con promedio de 37.,50 (+/- 11,81) años. La antigüedad en SO fue de 2 a 33 años, con promedio de 10,7 años. En la Tabla 1 se presentan los datos sociodemográficos de los participantes.

\section{Tabla 1.}

Datos sociodemográficos de médicos de Salud Ocupacional de empresas de México, 2014.

\begin{tabular}{lccccc} 
Médico & Sexo & $\begin{array}{c}\text { Edad } \\
\text { (años) }\end{array}$ & Edo. Civil & Escolaridad & $\begin{array}{c}\text { Antigüedad } \\
\text { (años) }\end{array}$ \\
\hline 1 & M & 26 & Soltero & Estudia posgrado & 2 \\
2 & F & 34 & Casado & Licenciatura & 8 \\
\hline 3 & F & 31 & Casado & Posgrado & 4 \\
4 & M & 26 & Soltero & Licenciatura & 2 \\
\hline 5 & M & 59 & Casado & Especialidad no terminada & 33 \\
6 & F & 30 & Unión libre & Licenciatura & 4 \\
\hline 7 & F & 53 & Casado & Licenciatura & 19 \\
8 & M & 54 & Casado & Estudia posgrado & 29 \\
9 & M & 28 & Casado & Posgrado & 3 \\
\hline 10 & F & 33 & Soltero & Posgrado & 6 \\
\hline
\end{tabular}

Fuente: directa. 
Para la recolección de datos se utilizaron entrevistas individuales en profundidad que, como expresa Aguilera-Velasco, siguen el modelo de una conversación pero difieren en que son una herramienta de investigación, una forma intencional de aprender acerca de los sentimientos, pensamientos y experiencias de la gente. Son conversaciones entre extraños guiadas por el investigador que realiza preguntas en profundidad, las cuales no han sido estructuradas previamente, sino que surgen en la dinámica que se genera entre entrevistado y entrevistador. ${ }^{21}$

Para entrevista se utilizaron preguntas abiertas, tales como, ¿Cómo es un día normal de trabajo para usted? ¿Por qué decidió dedicarse a la salud ocupacional? ¿Qué es para usted la salud ocupacional? ¿Le gusta su trabajo?

Todas las entrevistas se realizaron en una sola sesión y duraron entre 40 y 90 minutos. Fueron grabadas con teléfono celular, previa autorización de los participantes se transcribieron y almacenaron en computadora. Una vez convertidas a formato de tipo texto enriquecido (rtf) fueron procesadas como unidad hermenéutica con el sotware Atlas-ti. Con apoyo del mismo, se fueron seleccionando fragmentos significativos o citas de las diez entrevistas, codificando y generando familias o categorías.

Conforme se iban seleccionando citas y obteniendo códigos, se iban comparando y, dado el caso, fusionando con los códigos obtenidos previamente. Esta comparación se realizó de manera constante hasta observar que ya no surgían nuevos códigos, considerándose así que se había llegado a la saturación teórica.

Asimismo, con ayuda del programa Atlas.ti, se procedió a la estructuración esquemática de las redes teóricas de cada una de las familias.

Una vez desarrollados sistemáticamente los productos del análisis y explorados desde diversos ángulos y perspectivas, se constituyó un esquema lógico, sistemático y explicativo, integrando finalmente, la teoria. ${ }^{20}$

\section{RESULTADOS}

Mis motivos de elección, Cambié de vida, y Oportunidades y proyectos fueron las categorias emergentes de esta investigación, con las que se integró la dimensión de Adaptación al puesto de trabajo como elemento básico de la construcción teórica de la satisfacción laboral de los médicos de salud ocupacional. Se analizaron las percepciones de los médicos en estos tres momentos vitales de su vida laboral: inicio, desarrollo y como expectativa a futuro, considerando que la satisfacción laboral es una actitud que implica conocimientos y creencias que los trabajadores tienen con respecto a su trabajo. Los conocimientos que derivan de la experiencia del profesional médico en el ámbito empresarial. Y las creencias con respecto a su labor, que se fueron generando con sus experiencias, conforme "vivían y entendían", como mencionan Aguilera et al. ${ }^{15}$

Como indica la teoría fundamentada, se presentan las categorías emergentes con los respectivos códigos y citas textuales de los participantes que los generaron.

\section{Categoría 1: Mis motivos de elección}

Con respecto al porqué se encuentran trabajando en salud ocupacional, sólo algunos participantes refirieron haber elegido esta área desde el inicio de su vida laboral. Los más mencionaron que entraron a trabajar en la empresa por alguna circunstancia casual, ya que se les presentó la oportunidad o buscaron cubrir un vacío, por ejemplo, por no haber podido entrar a estudiar una especialidad médica clínica. Los códigos que fundamentan esta categoria con las respectivas citas textuales de los participantes, fueron:

Porque me llamó la atención

Los pocos participantes que mencionaron haber escogido la salud ocupacional como proyecto de vida desde el inicio expusieron entre sus motivos de elección que: "Me llamó mucho la atención el que un médico de salud ocupacional tiene que hacerla medio de ingeniero y medio de abogado y, aparte, de médico"; y "Me di cuenta que en medicina no se hace prevención; todo es curativo, todo es paliativo y la alternativa para hacer prevención es la salud ocupacional".

\section{Por una oportunidad}

En general, la salud ocupacional no fue mencionada como proyecto de vida. Surgió como alternativa como ellos refirieron: "Básicamente, fue una oportunidad. No lo decidi, se dio la oportunidad". También comentaron: "Nunca lo pensé, se me fue dando". 0 bien: "Yo caí de rebote, pero actualmente estoy fascinado con mi trabajo, me gusta muchísimo". Un participante aseguró que fue "Por azares del destino, porque la verdad a mí, cuando estaba en la escuela, no me llamaba mucho la atención, de hecho, me dieron muy poquito de medicina del trabajo". 0 también porque "Lo tomé como un trabajo alterno y rápido".

Algunos puntualizaron que fue "Por no pasar el examen nacional de residencias para la especialidad médica y... te quedas sin actividad, sin ingresos y pues, obviamente, uno tiene que pagar deudas y trabajar en algo". 0 porque "He visto compañeros que han sufrido porque no entran a la especialidad médica y duran un año llorando... e igual al otro año... y dices no, pues no es posible, y entonces pensé, bueno pues me puedo dedicar a esto, no está saturada el área y yo soy muy buena para organizar cosas y esto suena organizacional, ¿no?, y pues así fue como descubrí esta área que me gusta".

\section{Categoría 2: Cambié de vida}

La categoría cambié de vida se integró por códigos que expresan la percepción de su "experiencia" laboral, pues aun cuando no todos los participantes habían elegido como primera opción dedicarse a la salud ocupacional, para el momento del estudio (al menos tenían dos años de antigüedad) se encontraban adaptándose. Mencionaron que habían "cambiado sus objetivos de vida", "su actitud", "habian aprendido a delegar, a organizarse" y "a disfrutar el convivir con sus compañeros no médicos”. Los códigos que fundamentaron esta categoría con sus citas textuales fueron: Cambié de objetivos de vida

En este aspecto, comentaron "Cambié mis objetivos de vida y en lugar de pensar en ser cardiólogo o cirujano, o lo que sea, pensé en ser un especialista en mi sitio de trabajo, desarrollar todo lo que fuera necesario para resolverle los problemas y, entonces, me puse a estudiar".

Cambio de actitud

Como resultado de su estancia en las empresas, refirieron que "He cambiado mi actitud... mira que he cambiado porque si antes me hubiera pasado esto... no me hubiera aguantado".

Aprender a hacer otras cosas

Entre los cambios que se dieron en su proceso de adaptación a su labor como médicos de salud ocupacional, dijeron haber tenido que aprender aspectos diferentes de los que se les enseñaron en la Licenciatura de Medicina. Lo explicaron así: "Cuando yo ingresé a 
esto, no sabía nada, nada, nada. Yo agradezco mucho a las personas que en un principio se acercaron o me acerqué a ellos para empezar en lo de la salud ocupacional porque realmente en aquel entonces en la licenciatura de medicina, de salud ocupacional, lo que se veía sólo era epidemiología, mas no salud ocupacional como lo que es actualmente". Y comentaron: "Aprende uno a hacer otras cosas, al principio todo lo quiere hacer uno, pero ya después ya no, ya te organizas con mantenimiento... con todas las áreas y todo sale mejor, claro que siempre y cuando sepa uno dirigir, porque si no sabe uno dirigir, se va el barco a fondo" y "Si se sabe uno organizar en estas situaciones pues va a ser más digerible todo. Cuando uno empieza, es complicado, pero después, aprende uno a delegar".

Me acostumbro a trabajar con gente no médico

Hablaron de su cambio en cuanto a sus relaciones interpersonales, y refirieron: "Aquí, mis compañeros de trabajo son gente no médico, y al principio es raro porque te topas de venir de ambiente de hospital, pero ahora ya me acostumbré a trabajar con todo tipo de gente: abogados, de marketing, contadores, proveedores, extranjeros, y la verdad es que en un principio lo sufrí, pero ahora, lo disfruto".

\section{Categoría 3: Oportunidades y proyectos}

La categoría de oportunidades y proyectos se fundamentó en códigos relacionados con las oportunidades que vislumbraron para promocionarse y crecer en el área y sus proyectos a futuro, pero solo algunos participantes dijeron percibir esa oportunidad. Otros negaron toda posibilidad dentro de la empresa donde actualmente laboran. Y otros cuantos afirmaron que buscarán otras oportunidades.

Por otra parte, comentaron que en su crecimiento profesional y probabilidades de realizar sus proyectos se ven bloqueados si la empresa no tiene presupuesto.

Los proyectos personales mencionados fueron de tipo académico, tales como publicar, hacer maestría o doctorado. Y de tipo administrativo, como ser coordinadores o trabajar en empresas transnacionales.

Algunos cuantos señalaron perspectivas completamente diferentes, como la jubilación o el cambio a una especialidad médica.

Los códigos que integraron esta categoría, con sus respectivas citas textuales, fueron:

Oportunidad de promoción

Esta oportunidad no es algo que pueda existir en todas las empresas o que se dé fácilmente. Solo dos participantes afirmaron "Tengo una oportunidad de promoción por el movimiento de personal", y "El puesto siguiente sería el coordinador de médicos, pero ese se lo están dando al médico que tiene mayor antigüedad y yo debo cumplir, al menos, cinco años en el puesto, para ser candidato a coordinador".

No se puede ser algo más

Sobre la ausencia de oportunidades de promoción, explicaron: "Yo soy el jefe de medicina en la empresa, entonces no se puede ser algo más, puesto que únicamente es el servicio médico, el doctor y ya, no hay algo más arriba por parte del servicio médico"; y aseguraron: "Yo siento que la promoción no puede ser gran cosa". Buscar otras oportunidades

Por otra parte, cuando consideraron sus oportunidades a futuro, mencionaron que "Una vez que tenga mi título de maestro en ciencias en seguridad e higiene, pues bueno, entonces ya buscaré otras oportunidades de trabajo". 0, definitivamente, afirmaron que buscarían otras áreas de oportunidad: "Ahora hay como un estan- camiento, que ya no va conmigo... necesito hacer algo, entonces estoy viendo otras empresas, u otras áreas de oportunidad, donde pueda crecer".

\section{Tengo proyectos}

En cuanto a sus proyectos, tanto de superación académica como de puesto de trabajo, comentaron que deseaban "sacar provecho a su experiencia y publicar", "estudiar la maestría”, "llegar a ser coordinador y de ser posible hacer un doctorado", "Convertirme en asesor externo", "Trabajar en una trasnacional en una jefatura de seguridad, salud, medio ambiente o tal vez alguna gerencia”.

No me veo aquí toda la vida

A diferencia, hubo quien aseguró: "Me gusta; sin embargo, no me veo aquí toda la vida”.

Con estos datos, podemos elaborar los siguientes supuestos en la concepción teórica de la satisfacción laboral de los médicos participantes:

- La salud ocupacional no es considerada por los médicos que se dedican a ella como parte de un plan de vida que conciben desde que estudian la carrera de Medicina. Como afirman, llegaron a ella porque "cayeron de rebote" o "por azares del destino". Por lo tanto, la satisfacción laboral no es algo que se dé desde el inicio, se va dando. Es un proceso, no es algo estático.

- La satisfacción laboral como actitud positiva se va adquiriendo como resultado de la adquisición de nuevos conocimientos, habilidades y creencias.

- Finalmente, los médicos de salud ocupacional, una vez adaptados e involucrados, se enfocan a desarrollarse dentro de ella de manera sostenida en todas sus esferas: académica, social y personal.

\section{DISCUSIÓN}

La concepción teórica de la satisfacción laboral de los médicos de salud ocupacional participantes difiere de aquellas definiciones ${ }^{2}$ que mencionan que es un estado emocional positivo o placentero, ya que en estos médicos no es algo estático, no es un estado, es un proceso. No se da, se va adquiriendo conforme se adaptan al puesto de trabajo y cambia durante el transcurso de su vida laboral.

Por otra parte, los hallazgos concuerdan con Reed y Sanderson ${ }^{16}$ en que las personas se adaptan o ajustan al entorno o lo adaptan a sus necesidades individuales o para su satisfacción, a través de su ocupación. Este ajuste se revela en el discurso de los médicos participantes, cuando hablan de que cambiaron objetivos de vida, adquirieron nuevos conocimientos (en Salud Ocupacional, a organizarse, a delegar), cambiaron de actitud volviéndose más tolerantes, y aprendieron a convivir con compañeros no médicos. Asimismo, consideran sus perspectivas a futuro en el área.

También coinciden con lo afirmado por Abrahan ${ }^{14}$ que asegura que la satisfacción laboral se relaciona directamente con la experiencia de la persona y que esta experiencia se transforma en la percepción del trabajador. Y tal como afirman Aguilera et $\mathrm{al}^{15}$, los médicos de salud ocupacional configuraron lo que significa para ellos la salud ocupacional, lo cual cambió su percepción y actitud hacia la misma; esto es, la construcción teórica de su satisfacción laboral.

Por otra parte, les genera frustración valorar las escasas oportunidades que tienen de crecimiento o promoción dentro de la empresa en que están laborando. Sin embargo, esto no es privativo de este grupo laboral ya que, como podemos comprobar en los resultados 
de encuestas realizadas por la Online Career Center (OCC), compañía de bolsa de trabajo por internet, es similar a lo que sucede con todas las actividades laborales en México, donde cuatro de cada 10 empleados afirman que no creen poder crecer profesionalmente en la empresa donde se encuentran laborando, y sólo el $16 \%$ percibe claramente dicha oportunidad. ${ }^{22}$

Bajo otra perspectiva, se puede considerar que los médicos participantes se encuentren en transición hacia la llamada vinculación psicológica (en inglés, work engagement) reflejada en su cambio de actitud hacia el trabajo, que es como se define la satisfacción laboral. ${ }^{2}$

La vinculación psicología se define como un estado mental positivo relacionado con el trabajo, caracterizado por vigor, dedicación y absorción. El vigor y la dedicación son considerados como los opuestos de las dimensiones del burnout: agotamiento y cinismo o despersonalización. Y a la transición desde el agotamiento hasta el vigor, se le llama activación; mientras que a la que va desde el cinismo hasta la dedicación, se le llama identificación. ${ }^{23} \mathrm{Y}$ aun cuando no se considera que estos médicos hayan partido desde el estado negativo o burnout, según esta perspectiva, están en proceso hacia la vinculación psicológica, hacia la satisfacción laboral; en las transiciones de activación e identificación, dentro de lo que se ha llamado en este estudio, adaptación al puesto de trabajo.

Concluyendo, la satisfacción laboral en los médicos de salud ocupacional se presenta conforme se da su adaptación al puesto de trabajo, pues no existe desde el inicio de su vida laboral en el área, sino que se va generando conforme van aprendiendo y cambiando de actitud con respecto al trabajo; y, finalmente se manifiesta, ya que una vez adaptados e involucrados, se enfocan a desarrollarse dentro de la salud ocupacional de manera sostenida en todas sus esferas: personal, académica y social.

Finalmente, se recomienda realizar más estudios relacionados con el tema con una aproximación cualitativa. En este caso, la teoría fundamentada permitió validar el conocimiento ordinario y analizar el proceso de construcción teórica de la satisfacción laboral que tienen los médicos de salud ocupacional.
1. Comisión Internacional de Salud Ocupacional, CISO-ICOH. Código internacional de ética para los profesionales de la salud ocupacional [en línea]. Roma: CISO/ICOH; 2002 [citado sep 2015]. Disponible en http://www.bvsde.paho.org/ cursoa_epi/e/lecturas/mod6/codigo.pdf

2. Ramírez A. Servicios de salud ocupacional. An Fac Med. 2012; 73 (1):63- 69.

3. Engels $\mathrm{F}$. El papel del trabajo en la transformación del mono en hombre. México: Era; 1979.

4. Peiró JM. 2000. Psicología de la organización. Madrid: Universidad Nacional de Educación a Distancia; 2000.

5. Cantonnet ML, Iradi J, Larrea A, Aldasoro JC. Análisis de la satisfacción laboral de los arquitectos técnicos en el sector de la construcción de Comunidad Autónoma del País Vasco. Revista de la Construcción. 2011; 10 (2):16-25.

6. Spector P. Psicologia industrial y organizacional: investigación y práctica. México: El Manual Moderno; 2002.

7. Fernández E. Introducción a la gestión. Valencia: UPV; 2005.

8. Argüelles $L$, Quijano R, Sahuí J. Satisfacción en el trabajo como promotora de la calidad de vida laboral en empleados del sector turistico campechano. RIDE. 2013; (10): 1-19.

9. Díaz-Echenique MS, Stimolo MI, Caro NP. Satisfacción laboral y síndrome de desgaste laboral en enfermeros de hospitales públicos Córdoba-Argentina. Med Segur Trab. 2010; 56 (218):22-38.

10. Almaki $M$, Fitzgerald $G$, Clark $M$. Quality of work life among primary health care nurses in the Jazan region, Saudi Arabia: a cross sectional study. Hum Resour Health. 2012; 10:30 -35.

11. Borges $M$, Alves $M$, Regina P. Job satisfaction of nursing staff: integrative review. Rev. Latino-Am. Enfermagem [on line]. 2011 [cited mar 2014]; 19(4):1047-55. Disponible en: www.redalyc.org/pdf/2814/281421958026.pdf.

12. Delgado $D$, Inzulza $M$, Delgado F. Calidad de vida en el trabajo: profesionales de la salud de Clínica Río Blanco y Centro de Especialidades Médicas. Med Segur Trab [en línea]. 2012 [citado mar 2014]; 58(228):216-223. Disponible en: http://scielo.isciii.es/pdf/mesetra/v58n228/original3.pdf
13. Moreno $A$, Aranda $C$, Preciado $M$, Valencia S. Calidad de Vida Laboral en Trabajadores de la Salud, Tamaulipas, México, 2010. Cienc Trab. 2011; 13(39):11-16.

14. Abrahan MG, Contreras JM, Montoya S. Grado de satisfacción laboral y condiciones de trabajo. Una exploración cualitativa. Enseñanza e Investigación en Psicología. 2009; 14(1):105-118.

15. Aguilera-Velasco MA, Pérez JJ, Pozos B, Acosta M. Significados culturales del concepto de salud en el trabajo en profesionales en formación. Med Segur Trab. 2012; 58(228):224-236.

16. Reed KL, Sanderson SN. Concepts of Occupational Therapy. Baltimore: Lippincot Williams and Wilkins; 1992.

17. Navarro $E_{1}$ Roche $H$, Fuentes del Burgo J. ¿Sabemos con quién trabajamos? Estudio cualitativo sobre el trabajo y la satisfacción laboral de los oficiales de la edificación. XVI Congreso Internacional de Ingeniería de Proyectos 11- 13 Jul 2012; Valencia, España. Valencia: UPV; 2012 [citado mar 2015]. Disponible en: http://aeipro.com/files/congresos/2012valencia/CIIP12_0368_0378.3721.pdf

18. Cousins $R$, Donnell $C$. Nurse Prescribing in general practice: a qualitative study of job satisfaction and work - related stress. Fam Pract. 2011; 29: 223-227.

19. Gómez M. Estar ahí, al cuidado de un paciente con demencia. Invest Educ Enferm. 2007; 25(2):60-71.

20. Strauss A, Corbin J. Bases de la Investigación Cualitativa. Técnicas y procedimientos para desarrollar la teoria fundamentada. Medellin: Universidad de Antioquia; 2002.

21. Aguilera-Velasco MA. Salud y jubilación. Aproximación fenomenológica en profesores universitarios. Universidad de Guadalajara, Jalisco. México: Página Seis; 2010.

22. OCC. Indice OCC del empleo; Reportes 2012 [en línea]. Tlalnepantla de Baz: OCCMundial; 2012. [citado mar 2014]. Disponible en: http://empleoccmundial.com/

23. Salanova M, Llorens $\mathrm{S}$. Estado actual y retos futuros en el estudio del burnout. Papeles del Psicólogo. 2008; 29(1):59-67. 